\title{
Telisotuzumab Vedotin
}

National Cancer Institute

\section{Source}

National Cancer Institute. Telisotuzumab Vedotin. NCI Thesaurus. Code C118571.

An antibody-drug conjug ate (ADC) composed of telisotuzumab, a monoclonal antibody against the tumor-associated antigen (TAA) and proto-oncogene, c-Met receptor tyrosine kinase (c-Met; MET; hepatocyte growth factor receptor; HGFR) conjug ated to the cytotoxic agent monomethyl auristatin E (MMAE) via a valine-citrulline (vc) peptide linker (vc-MMAE; vedotin), with potential antineoplastic activity. Upon intravenous administration, the monoclonal antibody moiety of telisotuzumab vedotin targets and binds to c-Met expressed on tumor cells. Upon binding, internalization and enzymatic cleavage, the cytotoxic agent MMAE is released into the cytosol. MMAE binds to tubulin and inhibits tubulin polymerization, which results in G2/M phase arrest and tumor cell apoptosis. This kills the c-Met-expressing tumor cells. c-Met, a receptor tyrosine kinase overexpressed or mutated in many tumor cell types, plays key roles in tumor cell proliferation, survival, invasion, metastasis and tumor angiogenesis. 